In press: Nature Human Behavior

\title{
Homophily and Acrophily as Drivers of Political Segregation
}

\author{
Amit Goldenberg ${ }^{1^{*}}$ \\ Joseph M. Abruzzo ${ }^{2}$ \\ Zi Huang ${ }^{1}$ \\ Jonas Schöne ${ }^{3}$ \\ David Bailey ${ }^{1}$ \\ Robb Willer ${ }^{4}$ \\ Eran Halperin ${ }^{5}$ \\ James J. Gross ${ }^{6}$
}

\footnotetext{
${ }^{{ }^{1}}$ Harvard University, Harvard Business School, Cambridge, United States

${ }^{2}$ Northwestern University, Kellogg School of Management, Chicago, United States

${ }^{3}$ University of Oxford, Department of Experimental Psychology, Oxford, England

${ }^{4}$ Stanford University, Department of Sociology, Stanford, United States

${ }^{5}$ Hebrew University, Department of Psychology, Jerusalem, Israel

${ }^{6}$ Stanford University, Department of Psychology, Stanford United States
}

Corresponding Author: Correspondence concerning this article should be addressed to Amit Goldenberg. E-mail: agoldenberg@hbs.edu. 


\begin{abstract}
Political segregation is a significant social problem, increasing polarization and impeding effective governance. Prior work has viewed the central driver of segregation to be political homophily, the tendency to associate with others who have similar views. We propose that in addition to homophily, people's social tie decisions are driven by political acrophily, the tendency to associate with others who have more extreme political views (rather than more moderate). We examined this using a paradigm in which participants share emotions and attitudes on political policies, observe others' responses, and choose which others to affiliate with. In four studies ( $\mathrm{N}=1235)$, both liberal and conservative participants' social tie decisions reflected the presence of acrophily. We found that participants who viewed peers who expressed more extreme views as more prototypical of their political group also tended to engage in greater acrophily. These studies identify a previously overlooked tendency in tie formation.
\end{abstract}


The extreme levels of political segregation evident in the United States today presents a major social challenge ${ }^{1}$. Political segregation reduces interactions between group members, which contributes to polarization and intergroup hostility, and undermines political civility and effective governance ${ }^{2-4}$.

Prior work has focused mainly on one key driver of segregation, namely political homophily, defined as the tendency to affiliate with others with similar political views ${ }^{5-9}$. Political homophily is a pervasive and enduring propensity ${ }^{10}$, which results in the formation of echo chambers of like-minded people who rarely interact across political lines ${ }^{11,12}$. But does homophily alone drive individuals' decisions about who to affiliate with in political contexts?

In the current paper, we propose that in addition to political homophily, people's tie selection decisions are also driven by political acrophily, which we define as the tendency to prefer to affiliate with others who represent more extreme (as opposed to more moderate) political views in the direction of one's political leaning. Take for example a case in which a conservative selects between two conservative peers of the exact same distance from their view, one who is more extreme and the other is more moderate. The prediction of who is chosen based on homophily would suggest an equal probability to both ties, whereas a prediction based on acrophily would suggest a preference for the more extreme peer. Determining whether political acrophily is evident in addition to homophily is important because it could be a critical catalyst of political segregation in social networks, and by extension, a driver of attitudinal and affective polarization.

Homophily and acrophily are not mutually exclusive, and given the documented strength of political homophily, they are likely to co-occur. To illustrate how the two may 
integrate, consider a liberal who responds with outrage to an incident of police brutality and then encounters others' responses to the same incident. We illustrate three combinations of the two propensities (Figure 1): Under homophily, the chooser would affiliate with others whose outrage responses are closest to their own (Figure 1A), regardless of whether these responses show more or less outrage. Under homophily + acrophily (Figure 1B), the chooser would be asymmetrically biased towards the one side of the scale that is considered a more extreme view related to one's political beliefs, in this context others who express more outrage than they do. However, the chooser would not completely exclude others who expressed less outrage if their responses were similar. Finally, under acrophily, the chooser would elect to affiliate only with others who express more extreme responses than their own, which in this case is people who express more outrage (Figure $1 \mathrm{C}$ ). These strategies operate on a continuum, and dividing them into these three categories is done for heuristic purposes. Nevertheless, considering the strength of homophily, our assumption is that participants' choices should be most similar to the homophily+acrophily strategy.

Indirect evidence for its occurrence can be found in existing work. One relevant finding is from research on group deviance, which suggests that people evaluate deviation from group norms towards the extreme more favorably than deviations towards more moderate views ${ }^{13^{-16}}$. A second relevant finding is that people like others who present more coherent political views, a tendency which is often associated with more extreme views ${ }^{17^{-19}}$. Third, acrophily can be extrapolated from the notion of "directional voting" ${ }^{20-22}$, which suggests that people have a clear preference for representatives of their side, even if the distance in attitudes to these representatives is bigger than to representatives on the other 
side. One corollary of this idea is that people are attracted, to a certain extent, to representatives who are more extreme than them. However, this idea has been the focus of a great debate without clear empirical evidence for its occurrence ${ }^{23,24}$. Furthermore, it is not clear that preference for representatives on the one hand, and social ties, on the other hand, capture an identical phenomenon. Finally, a fourth indication of a tendency to prefer extreme views comes from the literature on attitude polarization ${ }^{25-31}$. One driver of polarization, among many, is people's tendency to express extreme views to be "better" than others in their group ${ }^{32-36}$. Interestingly, while the mechanisms that drive such polarization point to an attraction toward extreme views (as in acrophily), the individual-level immediate outcomes are actually opposite in the two cases. When polarizing, people adopt more extreme views, and therefore become more extreme in relation to others. Selecting ties based on acrophily, however, results in individuals becoming less extreme than others in their group because they have selectively affiliated with more extreme others. While acrophily may eventually lead to polarization, the process by which this occurs is completely different.

If acrophily were in fact robustly demonstrated, why might people prefer affiliating with more extreme group members? We suggest that one possibility is that more extreme views may be perceived as more prototypical of one's political group. People tend to believe their group holds more extreme views on average than they actually do ${ }^{37-41}$. If people estimate the views and emotional responses of their political group to be more extreme than they actually are, they may be more attracted to extreme compared to moderate views because they think that these views are more prototypical representations of their political group. 
We conducted four studies with the goal of examining the occurrence of acrophily. In Studies 1-3 we examined participants' tie selection strategies as they choose other participants based on others' responses of moral outrage to cases of police brutality. In Study 3 we show that one mechanism for acrophily is the perception that the prototypical view of the group is more extreme. Study 4 was designed to examine the occurrence of acrophily in a different context from emotions to police brutality, providing evidence of its existence when selecting social ties based on support for political policies.

\section{Results}

Analyses for all studies were conducted in $\mathrm{R}$ using mixed models for repeated measures. Assumptions of normality (Kolmogorov-Smirnov test) and equal variance (Levene test) were done for all main analyses (see sections on Formal Tests of Assumptions in SI for full report). When these assumptions were violated, we conducted a robust estimation of mixed effects using the package robustlmm ${ }^{42}$ finding similar results in all cases (see SI).

\section{Acrophily Based on Moral Outrage to Police Brutality}

The goal of Studies 1-3 was to examine participants' tie selection strategies when evaluating responses of moral outrage to pictures of police brutality against Black demonstrators. We chose to focus on expressions of outrage - defined as a negative emotional response, primarily consisting of anger, toward a transgressor in reaction to a wrongdoing ${ }^{43}$

- because it has been shown to be especially important in social signaling ${ }^{44,45}$ and in driving affiliation decisions ${ }^{18,46}$. We chose to specifically focus on responses to police brutality both because it is a highly salient issue and because responses to police brutality vary greatly by political affiliation in the U.S ${ }^{47}$. In Study 1 we examined liberals' tie selection decisions when 
choosing from a network of liberals and conservatives. In Study we 2 transitioned to an online design which allowed us to increase the sample size to include both Liberals and Conservatives. In Study 3 we replicated the findings of Study 2 and examined a potential mechanism for the effects, namely the tendency to evaluate the prototypical belief of one's group as more extreme. We had a few pre-registrations of hypotheses (Studies 1 and 2 were pre-registered; Study 1:https://osf.io/nguxy, Study 2: https://osf.io/xdnj4, see SI for review), the most central one was that participants' tie selection decisions would involve some degree of acrophily, such that participants' tie selection choices would be asymmetrically biased toward preferring responses that represented a more extreme view based on their political view. In the case of outrage to police brutality, acrophily for liberals meant preferring peers that expressed stronger outrage and acrophily for conservatives meant preferring peers that expressed weaker outrage.

Task. The task was similar in Studies 1-3, with small variations described in each study (Figure 2). In each trial, participants saw pictures of police brutality against Black demonstrators intended to elicit negative emotions. For each picture, participants were first asked to provide their emotional response to the picture in a few words and then to rate their emotions in response to the picture on a scale of 1 (no negative emotion) to 9 (a lot of negative emotion). After rating and responding to the picture, participants then saw six boxes with unique colors, each containing a peer response to the same picture that they just rated. Each box included a name that was congruent with the participant's gender, a peer rating, and their text response to the same picture. Peer ratings and responses were collected in a pre-test and included real ratings by real participants. After observing the responses for five 
seconds, participants' choices were different in the choice versus no choice conditions. In the choice condition, participants were asked to select three peers they would not want to see in a subsequent trial by clicking on the boxes they wished to eliminate. In the no choice condition, participants were asked to eliminate a random subset of three names colored in red. In both conditions, participants were told that the eliminated peers would be eliminated completely from the entire task and their names and responses would not be seen again, while the kept participants would be seen in the subsequent trial. We created the two conditions to examine the occurrence of homophily (which was found) and to examine potential differences in ratings as a result of tie selection (which were not found). The lack of difference in ratings allowed us to omit the control condition from Studies 3 and 4. See SI for full analysis. Participants completed the task for 20 trials (see methods for full description).

Studies 1 -2. Participants in Study 1 were self-identified liberals who completed the task in the lab. Participants in Study 2 were both liberals and conservatives who completed the study online with an effort to increase the sample size and diversity in political affiliation (compared to what could be found around Stanford). In both studies, participants were randomly divided into the choice and no choice conditions. This was mainly designed to provide evidence for the occurrence of homophily which was found in both studies, and to look at changes in ratings as a function of condition, which were not found (see Analysis of Participants' Emotions in SI). Our main analysis of acrophily focuses on results from the choice condition.

To examine the occurrence of acrophily we conducted two tests, both focusing on the choice condition. Our first test of acrophily was done by creating an acrophily coefficient for 
each participant in each trial and tested whether these coefficients were similar or different from zero, and whether they changed over time. Our acrophily coefficient was designed to estimate the difference between participants' actual choices and what would have been a symmetrical homophily choice. Recall that in each trial, participants were presented with ratings of 6 peers, and were asked to eliminate three peers (and keep three peers). Participants therefore had $\left(\begin{array}{l}6 \\ 3\end{array}\right)=20$ possible choices of sets of peers to keep in each trial. Of these 20 average combinations, one combination (or more) reflected the most homophilous choice in each set. In order to create our coefficient, we calculated a difference score between the most homophilous combination of potentially chosen peers, and the combination that participants actually chose. A positive number indicated that the combination of peers that participants chose to keep expressed stronger emotions on average than the most homophilous combination of peers, and the opposite for a negative number. Zero reflected no acrophily in participants' choice (see Acrophily Coefficient section in SI for mathematical representation). We standardized the acrophily coefficient by dividing it by the standard deviation of all possible choices of sets of peers.

In both Studies 1 and 2, we calculated an acrophily coefficient for each participant in each trial in the choice condition and we created a mixed-model analysis predicting the acrophily coefficient from trial number. We standardized trial number in order to be able to evaluate the average coefficient using the intercept of the model. In addition, and similar to the previous analysis, we used a random intercept of participants' id. Furthermore, in Study 2 we added an interaction term with political affiliation to evaluate differences in acrophily between the political groups. We report results by political affiliation although no significant 
differences were found between political groups (See Acrophily and Political Affiliation in SI). Notice that coefficients for all of our analysis are in standardized values and can be compared across studies.

For our liberal participants, results suggested that the acrophily coefficient was significantly positive (Study $1: b=.33, t(30)=2.51, p=.01, R^{2}=.23,95 \%$ Confidence Intervals $=[.07, .60]$; Study $2: b=.29, t(195)=3.56, p<.001, R^{2}=.32,95 \%$ Confidence Intervals $=[.11, .44]$ ), pointing to an acrophily. Furthermore, results of Study 2 (same direction to Study 1 with less power) suggested that liberal tendency for acrophily increased over time (Study 1: $b=.08, t(588)=1.49, p=.13, R^{2}=.23,95 \%$ Confidence Intervals $=[-.02$, $.19]$; Study 2: $b=.05[.001, .11], t(3760)=1.98, p=.04, R^{2}=.32$, Confidence Intervals $=[.02$, .05], see Figure 2A).

We then examined the same effects with our conservative participants in Study 2. Looking first at the intercept of the model, results suggested that the acrophily coefficient was negative and significantly different from zero $\left(b=-.21, t(196)=-2.53, p=.01, R^{2}=.32,95 \%\right.$ Confidence Intervals $=[-.40,-.06])$, pointing to an acrophily tie selection strategy toward a less emotional response. Unlike Study 1 (and Study 3) we did not find a change in conservatives acrophily coefficient over time $\left(b=.02, t(3760)=0.73, p=.46, R^{2}=.32,95 \%\right.$ Confidence Intervals $=[-.03, .03])$.

While our first test of acrophily was able to show a difference in tie selection relative to a homophilous, balanced choice, we could not compare the degree of acrophily seen by participants to other strategies. Our second test of acrophily was designed to address this limitation by comparing participants' actual tie selection choices in the choice condition to 
what they would have chosen if they used four different tie selection strategies: a no choice strategy, homophily (without any acrophily), and two complete acrophily strategies (one toward more emotional ratings, and one toward less emotional ratings). Simulating participants' tie selection choice in these strategies was done by using participants' actual ratings to the pictures they saw, but instead of making the actual tie selection choices that they made, our simulation executed one of the four tie selection strategies mentioned above. Notice that trials in our task are dependent, such that the peers that participants saw in a given trial were dependent on their choices in previous trials. Therefore, our simulation was designed to replicate the task structure from the first trial and allow the simulation choices in each trial to affect the peers in the following trial.

In the random selection strategy, agents in our simulations chose peers randomly. In the homophily strategy, agents in our simulations chose in each trial the three peers closest to participants' ratings, regardless of whether they were more moderate or more extreme. In both of our acrophily simulations (more outrage and less outrage), agents in our simulations chose the peers closest to their own rating, but only on one side of the scale, either more outrage or less outrage. Only if there were no available peers in designated side (more or less depending on the simulation) agents chose the closest peer from the other side.

We ran 1000 iterations of each of the four simulations (random selection, homophily, acrophily higher, acrophily lower) such that each simulation generated an average of rating of the kept peer in each trial. We then ran 1000 models in which we compared participants' actual average kept ratings (the peers that participants decided to keep) to the average rating of the peers that were kept in each of the four simulations. Each analysis was done using a 
mixed-model analysis comparing between the actual average kept ratings by each participant, and the average kept ratings of each of our four simulations in the same model. We also included a by-participant random intercept in each model. We report here the $b$ and $t$ values for each comparison between the actual kept ratings and one of the simulations with a 95\% confidence interval for $t$ values and $p$ values when it is necessary (Figure $3 \mathrm{~B}$ ).

Looking first at the liberal participants, results suggested that the average rating of the actually kept peers was significantly higher than the homophily simulation (Study $1: b=-$ $.646, t=-3.58,95 \%$ Confidence Intervals $=[-.650,-.643], p<.001 ;$ Study $2: b=-.27, t=-2.60$ $95 \%$ Confidence Intervals $=[-.275,-.273], p=.010[.010, .11])$, and significantly lower from the more outrage acrophily strategy in Study 2 but not in Study 1 (Study 1: $b=.327, t=1.82$, $95 \%$ Confidence Intervals $=[.323, .330], p=.088[.084,0.92] ;$ Study $2: b=.396, t=3.75$, $95 \%$ Confidence Intervals $=[.395, .397], p<.001)$, indicating that participants' tie selection strategy was between homophily and acrophily in Study 2 and marginally significantly different from acrophily in Study 1. Coefficient sizes indicate that differences from acrophily high was $\sim 50 \%$ smaller in Study 1 and $44 \%$ bigger in Study 2 than the distance to homophily. These findings suggest that despite the fact that participants' choices in both studies fell between homophily and acrophily, there was quite a lot of variance in whether participants behavior was closer to homophily or acrophily. Finally, as expected, the average kept ratings in participants' actual choice was also significantly higher than a random selection (Study $1: b$ $=-1.111, t=-6.15,95 \%$ Confidence Intervals $=[-1.117,-1.106], p<.001$; Study $2:: b=-1.313, t=-$ 12.44, 95\% Confidence Intervals $=[-1.316,-1.311], p<.001)$ and the acrophily lower strategy (Study 1: $b=-1.911, t=-10.58,95 \%$ Confidence Intervals $=[-1.915,-1.907], p<.001$; Study $2: b$ 
$=-1.351, t=-12.80,95 \%$ Confidence Intervals $=[-1.353,-1.350], p<.001)$. Results from our comparisons in the choice condition provided further evidence that participants' tie selection choice included a combination of homophily + acrophily toward more emotional responses.

Looking next at the conservative participants in Study 2, as expected, results suggested the exact opposite: conservatives peer choices were lower than what they would have chosen with just homophily $(b=.271, t=2.02,95 \%$ Confidence Intervals $=[.269, .272]$, $p=.04[.044, .45])$ and higher than the acrophily lower simulation $(b=.515, t=-3.85,95 \%$ Confidence Intervals $=[-.517,-.514], p<.001)$, suggesting that choices were in between homophily and acrophily lower (but $88 \%$ closer to homophily in this case), which represented a more extreme conservative view. As expected, the average rating of the actually kept peers was significantly lower than a random selection $(b=.860, t=6.43,95 \%$ Confidence Intervals $=[.857, .862], p<.001)$ and also significantly lower than the acrophily higher simulation $(b=1.192, t=8.91,95 \%$ Confidence Intervals $=[1.190,1.194], p<.001)$. Results from our comparisons in the choice condition provide further evidence that participants' tie selection choices involved a combination of homophily and acrophily, but in this case toward less intense ratings.

Study 3. The goal of Study 3 was to replicate findings of Study 2 and further test a potential mechanism driving acrophily. We hypothesized that participants were selecting ties who are more extreme because they evaluated these extreme responses as more prototypical of their groups' responses. To evaluate this hypothesis, participants first completed a task that was similar to that of Studies 1 and 2 (with some minor differences, see Methods). At a second stage, participants were then shown six of the trials they completed again (with the 
exact same peer responses) and were asked to rank the three responses that represented the most prototypical response of their own political group. We did not provide participants further details regarding the meaning of that term but assumed that participants understood it to be related to the political spectrum.

Similar to Studies 1 and 2, we first found evidence for homophily (see Homophily Analysis in SI). We then tested whether participants' tie selections were also driven by acrophily. Looking first at the acrophily coefficients using the same analysis as in Studies 1 and 2, results suggested that the coefficient for the liberal participants was positive, significantly different from zero and very similar to the one found in previous studies $(b=.32$, $t(374)=6.54, p<.001, R^{2}=.28,95 \%$ Confidence Intervals $\left.=[.22, .42]\right)$. Similar to Studies 1 and 2, the acrophily coefficient became stronger with trial number for liberal participants $(b$ $=.04, t(7161)=2.46, p=.01, R^{2}=.28,95 \%$ Confidence Intervals $\left.=[.01, .08]\right)$. For the conservative participants, results suggested that the acrophily coefficient was negative, significantly different from zero, and slightly smaller than in Study $2(b=-.15, t(374)=-3.06$, $p=.001, R^{2}=.28,95 \%$ Confidence Intervals $\left.=[-.25,-.05]\right)$, pointing to an acrophily tie selection strategy. Interestingly, results suggested that the acrophily shown in our conservative participants decreased with trial number $\left(b=.06, t(7161)=3.46, p<.001, R^{2}=\right.$ $.28,95 \%$ Confidence Intervals $=[.03, .10])$. It is unclear why acrophily decreased for conservatives and increased for liberals. This could be caused by the salience of strong emotional responses that reduce the strength of acrophily over time, or by some inherent differences in the way acrophily operates between liberals and conservatives. 
We then turned to our second method of evaluating acrophily, by comparing it to simulated data of various strategies replicating the results of Study 2. Looking first at liberals, results suggested that the average rating of the actually kept peers was significantly higher than just homophily $(b=-4.87, t=-5.06,95 \%$ Confidence Intervals $=[-4.86,-4.89], p<.001)$, and significantly lower compared to the acrophily higher simulation ( $b=5.88, t=6.11,95 \%$ Confidence Intervals $=[5.87,5.89], p<.001)$, revealing again a mix of homophily and acrophily, with distant from acrophily $\sim 20 \%$ bigger. Finally, as expected, the average kept rating in participants' actual selection was significantly higher than a random selection $(b=-$ 15.22, $t=-15.81,95 \%$ Confidence Intervals $=[-15.24,-15.20], p<.001)$, and also significantly higher than the acrophily lower simulation $(b=-19.26, t=-20.00,95 \%$ Confidence Intervals $=$ $[-19.27,-19.25], p<.001)$, suggesting that average kept ratings are between homophily and acrophily. Findings of the conservative participants were as expected, the exact opposite. The average rating of the conservative tie selection was lower than just homophily $(b=2.28, t=$ $2.28,95 \%$ Confidence Intervals $=[2.27,2.29], p<.001)$, and higher than the acrophily lower simulation $(b=-7.24, t=-7.25,95 \%$ Confidence Intervals $=[-7.25,7.23], p<.001)$. In this case, however, conservative choices were much closer to homophily that previously seen (although still significant on average). Finally, as expected, the average kept rating in participants' actual choices was also significantly lower than a random selection ( $t=\mathbf{2 1 . 2 6}$, $t=21.29,95 \%$ Confidence Intervals $=[21.24,21.29], p<.001)$ and significantly lower than the acrophily higher simulation $(b=18.47, t=18.49,95 \%$ Confidence Intervals $=[18.46,18.48], p$ $<.001)$. 
After establishing the existence of acrophily, we then turned to examine one possible mechanism for acrophily. As a reminder, after completing the first phase of the task, participants were shown 6 previous trials that were identical to those they just saw and were asked to rank the most prototypical response of their group of the available 6 peers, from 1-3. To analyze this phase of the task, we created a difference score between participants' ratings and the peers participants marked as prototypical, such that a positive number indicated that the chosen prototypical person that was chosen by our participant rated the pictures more strongly than the participant and the opposite for a negative number. As participants chose prototypical peers in order (1-3), we did this for each of the three peers chosen by participants. We then conducted an interaction between the rank of prototypical peer (1-3) and participants' political affiliation in predicting the difference score between participants and the prototypical peer. The outcome could tell us whether the first selected prototypical peer was higher or lower than participant's rating (the intercept of the model), whether this difference was reduced for the second or third selected peer (slope), and whether this was different between liberals and conservatives (interaction).

In order to evaluate the difference from the most prototypical peer chosen by participants, we made sure that the intercept of the model was equal to the first peer. We first centered our model on the liberal participants. The intercept of the model was significantly positive, suggesting that the most prototypical peer designated by the liberal participants was estimated to express more negative emotions compared to participants' own ratings by 8.07 points on the $1-100$ scale $\left(b=8.07, t(545)=6.02, p<.001, R^{2}=.30,95 \%\right.$ Confidence Intervals $=[5.45,10.70])$. As expected, going down the prototypicality rank led to 
reduced difference between participants and the prototypical peer $(b=-4.15, t(545)=-7 \cdot 50, p$ $<.001, R^{2}=.30,95 \%$ Confidence Intervals $\left.=[-5.25,-3.07]\right)$, as fewer peers who expressed stronger emotions were available (see Figure 4). We then centered the model on the conservative participants. The intercept of the model was significantly negative, suggesting that the most prototypical peer designated by the conservative participants was estimated to express less negative emotions compared to participants' own ratings by 4.47 points $(b=-$ $4.47, t(545)=-3.23, p<.001, R^{2}=.30,95 \%$ Confidence Intervals $\left.=[-7.17,-1.76]\right)$. As expected, going down the prototypicality rank led to reduced difference between participants and the prototypical peer $\left(b=.56, t(545)=6.23, p<.001, R^{2}=.30,95 \%\right.$ Confidence Intervals $=[2.44,4.68])$. Notice that conservatives' third choice was on average higher in rating than participants' own ratings. This is likely caused by the fact that on average there weren't enough conservatives who were more extreme than participants by the third choice. Results also revealed a stronger difference for liberals, such that liberals assumed prototypical members to be more extreme compared to the assumption made by conservative (see Prototypicality and Political Affiliation in SI for further information).

Finally, to further investigate participants' evaluation of prototypical peers as a potential mechanism for acrophily, we examined the association between two tendencies: participants' degree of acrophily and their tendency to mark more extreme participants as prototypical. Given that the acrophily tendency and the ratings of prototypicality were done in different trials, we decided to average participants' acrophily coefficient as well as participants' average rating of the prototypical peer. Results suggested a significant association between participants' acrophily and participants' choice of the prototypical 
members of their political group $\left(b=.30, t(375)=6.29, p<.001, r^{2}=.09,95 \%\right.$ Confidence Intervals $=[.34, .44])$, supporting the idea that participants choice of prototypicality was associated with their degree of acrophily.

Study 4. The goal of Study 4 was to address key limitations of Studies 1-3. First, we wanted to examine whether acrophily can be seen in tie selection based on other types of responses than emotions, asking participants to rate and choose ties based on support for four different political policies associated with four highly contentious topics: affirmative action, gun control, hunting, and military budget. Second, we wanted to see whether acrophily is evident when participants choose to keep, rather than eliminate peers. Third, we wanted to simplify the task to allow acrophily to be detected without using simulations. In order to be able to achieve these goals, Study 4 has quite a different design from the previous studies. However, we believe that by addressing the limitations of Studies 1-3, we were able expand the notion of acrophily in important ways. In the revised task, participants provided text responses and rating of support (1-7 scale) for four political policies. After rating each policy, participants saw 7 peers who provided all potential responses to the policy on a 1-7 scale. Then, participants were asked to choose one peer whose response they would like to keep (see figure 5).

To evaluate acrophily, we created an acrophily difference score such that higher scores always indicated choosing to keep a peer whose rating represented a more extreme view in relation to the specific policy and political affiliation of the chooser. This allowed us to compare acrophily among participants with different political affiliations. We then conducted an analysis with participants' political affiliation as the independent variable and the 
acrophily difference score as the dependent variable. The model also included two random intercepts, one of participants' id and one of policy type. Looking first at the intercept of the model, which was our liberal participants, results suggested that our liberal participants chose to keep peers whose rating represented a more extreme view compared to their own ( $b$ $=.12, t(427)=3.62, p<.001, R^{2}=.02,95 \%$ Confidence Intervals $\left.=[.05, .18]\right)$. There was no difference between liberals and conservatives in their degree of acrophily $(b=.01, t(415)=$ o.19, $p=.84, R^{2}=.02,95 \%$ Confidence Intervals $\left.=[-.08, .10]\right)$ as conservatives also showed a tendency for acrophily. Results provide important additional evidence regarding acrophily. First, it seems that acrophily can occur in context other than emotions. This is important because it suggests that acrophily is even more common than initially though and can occur when choosing ties based on a variety of response types. Second, it suggests that acrophily can occur when people choose new ties, rather than eliminate existing ones, again expanding the situations in which acrophily can occur. Third, the simplicity of the current task provides evidence that acrophily can occur in a much simpler contexts, in which participant do not need to make decisions on multiple ties at the same time. This is encouraging because it presents a road map for future research on this topic. Importantly, the effect that were found in this final study are relatively small, and much smaller for those in the previous studies. However, notice that this analysis includes participants who rated certain stimuli as 7 or 1 and therefore cannot reach acrophily. Removing such ratings (which represent 31.3\% of all ratings) doubles the effect (see Study 4 Analysis in SI). Albeit small, these results are especially striking given the structure of the task and the fact that participants just rated their 
response to the stimuli and then immediately chose users whose ratings are more extreme than their own.

\section{General Discussion}

The goal of the current project was to introduce the concept of acrophily - the tendency to prefer to affiliate with others with more extreme (as opposed to more moderate) political views - and to assess whether acrophily is evident in tie selection decisions as an additional driving force of political segregation and polarization. In four studies, we showed that participants tended to keep ties whose ratings reflected a preference toward more extreme (versus more moderate) views. Importantly, we found strong evidence for homophily as well, and participants' tie selection strategy seemed to reflect a mix of homophily and acrophily.

Our findings have important implications for understanding political segregation. If people's tie selection is affected by acrophily in addition to homophily, we should assume that segregation occurs at a faster rate and leads to more extreme outcomes than would otherwise be expected. To evaluate this possibility, we created an agent based model (see section on Agent-Based Model of Network Segregation in SI) that randomly populated a network of both liberals and conservatives and examined the rate and degree of network segregation as a result of homophily alone, acrophily alone, or a combination of homophily and acrophily similar to that found in our studies. As expected, our simulation suggested stronger segregation in either version featuring acrophily, relative to homophily alone.

Research on acrophily is especially important in the current digital era, in which people make many tie selection decisions every day. Social media algorithms are often 
designed to increase or decrease strength in ties based on users' activity. Liking a certain post on social media is likely to lead to an increase in tie strength with the person who produced the post, and ignoring a post is likely to contribute to weakening of a social tie. Therefore, in the many hours that people spend on social media everyday they make many tie selection decisions. Further specifying tie selection strategies and the psychological processes that give rise to them is of utmost importance in light of their daily prevalence and their impact on social segregation.

In addition to providing evidence for the existence of acrophily, results of Study 3 also provided an indication of why acrophily may be occurring, suggesting that one reason for the preference for more extreme views is that participants see the prototypical group member as more extreme than they actually are, and may want to have ties to these prototypical ingroup members. Further analysis supporting this idea revealed that individual-level tendencies to evaluate prototypical peers as extremes were associated with increased tendencies for acrophily. One limitation of the current measure is that participants were asked to indicated a prototypical member of their group, without specifically indicating a political group. Future work should reexamine this evaluation with a more specific indication of political group.

Despite this encouraging evidence, it is likely that other mechanisms also contribute to acrophily. One such mechanism is the relative salience of extreme views. Such salience may be caused by increased certainty ${ }^{48,49}$, the use of more covert signals of one's own group membership ${ }^{50}$, and the fact that increased certainty is likely associated with increased willingness to express political views. In the current set of studies, acrophily was found while keeping the frequency of occurrence constant. Yet, in real social interactions, extreme users 
may be even more salient, which should increase the effect of acrophily. Further studies should further examine these potential mechanisms looking at more natural interactions.

Our experiments leave open several questions regarding how acrophily is translated to emotions, attitudes, and behavior outside the lab. One limitation relates to the fact that in each study participants chose ties based on peers' responses to a single issue, whereas in the real world multiple issues drive processes of tie selections. We chose to minimize the complexity of information about the peers that participants saw in order to reveal the occurrence of acrophily. However, it is likely that tie selection decisions that are made based on responses to a single issue are more susceptible to acrophily than more complex tie selection decisions, in which people need to balance multiple issues ${ }^{51}$. Future studies should examine the existence of acrophily in natural social settings, e.g., on social media, or in closed organizations such as companies and schools. Future studies should also examine whether acrophily occurs in topics which are not political in nature.

A second limitation relates to the goals that participants had for keeping certain peers. In the current study, the goal was much simpler than in many interactions in real life: to keep seeing their response in a subsequent trial. In many situations outside the lab, people choose ties to achieve various affiliative and strategic needs. Future studies should modify the goals for participants' interactions and examine how they affect the occurrence of acrophily.

A third limitation of the current work is that we focused on providing evidence for acrophily rather than thinking about what could mitigate the effects of acrophily. Future work should examine the possibility of mitigating participants' tendency toward acrophily by 
either making them aware of such tendency, or by providing them information of the true prototypical ratings of their group members.

Finally, our findings raise interesting questions about the drivers of recent changes in political segregation. Given that we see much higher levels of segregation and polarization in the US and in other societies around the globe now than ever before, one interesting question is what has changed. A related question is what the future holds given the tendency of acrophily. It's possible that acrophily tendencies would taper off when segregation reaches an extreme level, but whether and when this would happen is not clear. We believe that our demonstration of the presence of both homophily and acrophily in political tie selection decisions can inform investigation of segregation and we see great potential in not only further investigating acrophily but also perhaps finding ways to reduce acrophily and segregation.

\section{Methods}

This research was approved by Stanford University (Study 1: IRB7373) and Harvard University (Studies 2-3: IRB19-1409, Study 4: IRB21-0698). All participants provided informed consent and were compensated for their time.

\section{Studies 1-3}

Participants. In Study 1, we used data from a task that resembled the task used in the present research in order to be able to estimate the required sample size. Our estimated sample size for Study 1 was based on finding evidence for homophily. Results suggested that using 30 participants completing 20 trials in our experimental condition would be enough to obtain almost 100\% power for the study (see SI for power estimations based on each sample 
size). As our study included both choice and no choice conditions, the final sample of Study 1 included 70 participants (Males: 26, Females: 42, Other: 2; Age: $\mathrm{M}=21.30, \mathrm{SD}=9.20$ ) randomly assigned either to a choice condition or to a no choice condition (described in detail below). All of the participants were American citizens who self-identified as liberals. Participants were recruited using the Stanford University paid participant pool, which includes a mix of Stanford students and community members. Participants received $\$ 10$ for their participation in the study. Notice that this lab study was merely the first examination of the phenomenon and sample sizes were dramatically increased in Studies 2 and 3 when transitioning the task to online studies. No participants who completed the study were removed from the analysis.

Study 2 was designed with the goal to recruit a larger and more diverse participant sample. For this reason, we converted our lab task into an online task and increased the target sample size to 400 participants (200 in the choice condition), with the hope of recruiting 200 liberals and 200 conservatives and increasing the power to almost 100\% (see Power Analysis in SI). Participants were recruited using the online platform Prolific in exchange for $\$ 2.50$ ( $\sim 20$ minutes). Out of the 400 participants who completed our task, we removed 8 participants for providing nonsensical text responses or not providing their demographics. Our final sample therefore included 392 participants (Males: 228, Females: 16o, Other: 4; Age: $\mathrm{M}=37.15, \mathrm{SD}=13.49$ ), who were randomly divided into no choice and choice conditions. All participants were American citizens. We were aiming to get an equal number of participants in each political side. Accordingly, in terms of their political affiliation, 
184 participants identified as liberals, 180 identified as conservatives, and 28 identified as located in the middle. We removed middle participants from the main analysis.

In Study 3, as in Study 2, we recruited 400 participants with the hope of recruiting 200 liberals and 200 conservatives. Participants were recruited using the online platform Prolific in exchange for $\$ 3.5$ ( $\sim 30$ minutes). Out of the 400 participants who completed our task, we removed 13 participants for providing nonsensical text responses or not providing their demographics. Our final sample therefore included 387 participants (Males: 236, Females: 149, Other: 2; Age: $M=43.25, S D=13.14$ ). All participants were American citizens. In terms of their political affiliation, 194 participants identified as liberals, 183 identified as conservatives, and 10 identified as located in the middle. Similar to Study 2, we removed middle participants from the main analysis.

Tie Selection Task. The structure of the tie selection task was the same in all studies with a few modifications which will be described below. Participants were told that they were taking part in a study with the goal of understanding how people respond emotionally to political situations, as well as how they make decisions about what responses they would like to see. The task included 3 practice (in which participants saw anger-inducing pictures relating to driving) and 20 actual trials. In the actual trials, participants saw pictures of police brutality against Black demonstrators. Pictures were pre-tested to elicit negative emotions, primarily anger (see full description in the Image Pool below). For each picture, participants were first asked to provide their emotional response to the picture in a few words (Figure 2). After providing their response, participants were asked to rate their emotions in response to the picture on a scale of 1 (no negative emotion) to 9 (a lot of negative emotion). We chose to 
use a neutral to negative emotion scale rather than a positive to negative scale for two reasons. First, measuring tie decisions based on a neutral to negative scale allowed us to test in Study 2 whether acrophily is caused by just attraction to more emotion, or rather to extreme views expressed by either no emotion or a lot of emotion, depending on one's political beliefs. Second, we specifically selected pictures that were evaluated as eliciting negative emotions by more than 90\% of the participants including conservatives (see description below), which means that positive raters would be outliers and would change the focus of the task.

After rating and responding to the picture, participants then saw six boxes, each containing a peer response to the same picture that they just rated. Each box included a name that was congruent with the participant's gender. Names were chosen randomly from a list of popular names from 2017 (see SI for full list). Below the peer name, participants saw the peer rating and their text response to the same picture. Each box had a unique color to help participants identify the peer in future trials. Peer ratings and responses were collected in a pre-test and reflected real ratings by real participants (see Image and Peer Pool for further details). Participants were forced to look at the 6 responses for 5 seconds before making any decisions. After 5 seconds, participants' choices were different based on whether they were assigned to the choice versus no choice conditions. In the choice condition, participants were asked to select three peers they would not want to see in a subsequent trial by clicking on the boxes they wished to eliminate. Clicking on each box eliminated it from the screen. We chose to instruct participants to eliminate those they didn't want to see rather than selecting those they did want to see in order to emphasize the fact that eliminated participants are never 
shown again. In the no choice condition, a random subset of three names was colored in red. Participants were asked to click on the boxes with the red names. In both conditions, participants were told that the eliminated peers would be eliminated completely from the entire task and their names and responses would not be seen again, while the kept participants would be seen in the subsequent trial.

After eliminating three peers in each trial, participants transitioned to the next trial, in which they were again asked to provide a text response and rate their emotional response to a new picture of police brutality. Participants were then shown 6 boxes with 6 responses to the new picture. In every trial except for the first, three of the six boxes were of peers who were kept from the previous trial. These boxes retained the same location, color, and name from the previous trial, and the peer ratings and responses were produced by the same original person who participants kept from the previous trial. The other three boxes were randomly generated from the peer pool and included new names, ratings, and responses. After completing 20 trials in which they provided their responses to new pictures and chose to eliminate three new peers, participants completed a short survey described below.

Image and Peer Pool. We conducted a pilot study to create the image pool for the study, testing 40 candidate pictures of police brutality against Black citizens. One hundred and four participants were presented with each picture and were asked to first provide a text response to the picture. Participants were then asked to rate their emotional response to the picture using the same scale that was used in the task. Finally, participants were asked to select whether observing the picture elicited a negative emotion, positive emotion, or no emotion at all. This question did not appear in the actual task and was used to select which 
pictures would be used. Two criteria were used to select the pictures. First, all of the selected pictures were rated by fewer than $10 \%$ of participants as positive. Second, we excluded pictures whose average rating was higher than 7 or lower than 3 (to allow variance in peer responses). As expected, our pictures were rated as eliciting significantly more negative emotion by liberals compared to conservatives (see Supplementary Figure 1 in SI).

Out of the 104 participants who completed the pilot study, we chose 63 participants to be part of our peer pool to allow for the elimination of three peers in each trial in addition to six peers in the first trial $\left(19^{*} 3+6\right)$. Our first criterion in choosing peers was to make sure that our peer pool was heterogeneous in terms of political affiliation. We chose 30 conservative, 30 liberals, and 3 participants who self-identified as middle. Our second criterion for choosing our peers was participants' text. We chose participants who mostly provided more than one-word responses to all of the pictures, and ones whose text responses were more or less similar to their ratings in terms of emotional intensity. After choosing our peer pool, we provided final editing of participants' responses to make sure that the peer text was coherent, and that it matched their ratings of the pictures. In Study 2 we slightly modified our peer pool by removing some liberal peers who provided less elaborate text, and substituting them with data from participants from Study 1 . Similar to Study 1, our final peer sample included 30 conservatives, 30 liberals and 3 peers who identified as being in the middle of the political spectrum.

Study 3 was based on Study 2's procedure with four meaningful changes. First, participants were only assigned to the select condition, as the goal was to get a better sense of the mechanism for acrophily. Second, we recreated our peer pool from that of Study 2, by 
employing a new set of peers who provided new responses to the pictures of police brutality in the task. The reason for this change is that the social movement related to the death of George Floyd occurred between Studies 2 and 3 and we suspected that such a large-scale movement may have changed people's attitudes toward police brutality. We therefore ran an additional pilot in which we created a new peer pool to the same pictures of Studies 1-2 (see SI). Third, we changed the scale participants used to rate their emotions from 1-9 to o-10o, o indicating no outrage and 100 very strong outrage. This was done to increase the variance in participants' responses. The fourth and most important change to the task was our measurements of the prototypicality of response. After completing the task in a similar way to Studies 1 and 2, participants then completed six additional trials in which they saw a copy of a previous trial (trials $3,6,9,12,15,18$ ) and were asked to rank the responses that represented the top three most prototypical responses of their political group using this question: "Choose the three peers that represent the most prototypical response of your political group.” After completing these six trials, participants were forwarded to a survey that included a few final questions described below.

Measures. While completing the task, we collected participants' ratings and text in response to each picture. We also recorded which peers they chose to keep versus eliminate. In Study 1, after completing the task, participants completed a survey which included two questions relating to their motivation to keep certain peers based on similarity and difference. In the similarity question participants were asked: "To what extent did you choose people who felt similar emotions to you in response to the pictures?" In the difference question participants were asked the same question, but using the words "different emotions" 
instead. Participants rated their response using a scale from 1 (not at all) to 6 (very much so). Participants also completed a few scales including a group identification scale ${ }^{52}, \mathrm{a}$ heterogeneity test in which participants were asked to estimate what portion of their social environment has different race, socio-economic status, a political affiliation measure, a need to belong scale ${ }^{53}$, and a personality scale ${ }^{54}$. These scales were measured with the intention of testing them as potential mechanisms for acrophily (See Supplementary Tables 1, 5-6, 10-11 in SI for full description and analysis of the connection between these scales and acrophily). In Study 2 we removed the need to belong scales from our survey and added a few new scales in which we examined potential motivations for acrophily (choosing people because they are more creative, provided new interpretations, made the participants feel good, etc.), feeling thermometers for various groups ${ }^{55}$, as well as participants' political identification (see SI for further analysis). In Study 3, after participants completed the task, we measured participants' ranking of peers' prototypicality using the task described above. Finally, participants answered a few survey questions that were similar to Study 2 (see SI).

\section{Study 4}

We recruited 400 participants after processing results from Study 3 to ensure that a sample of this size would produce power of $>.9$, even when we reduced the stimuli from 20 to 4. Participants were recruited using the online platform mTurk in exchange for \$3.5 ( 30 minutes). Out of the 400 participants who completed our task, we removed 14 participants for providing nonsensical text responses or not providing their demographics. Our final sample therefore included 386 participants (Males: 199, Females: 183, Other: 4; Age: M= 39.88, SD = 12.04). All participants were American citizens. In terms of their political 
affiliation, 217 participants identified as liberals, 169 identified as conservatives. We had no participants identified as middle in this study as participants had to make a binary choice at the beginning of the task.

Tie Selection Task. The structure of the task was based on the task used in Studies 13 with a few important modifications. The task included 1 practice trial and 4 actual trials. Participants saw texts describing a political policy (see description below). For each policy, participants were first asked to provide their text response to the policy in a few words. After providing their response, participants were asked to rate their support for the policy on a scale of 1 (completely against) to 7 (completely support).

After rating and responding to the policies, participants then saw seven boxes, each containing a peer response to the same policy that they just rated. While the type of content in the boxes was similar to Studies 1-3 and included a name, a rating, and a text, there were a few important differences in this stage compared to the task used in Studies 1-3. First, we made sure that all seven optional responses were present at each trial, covering the whole scale from 1 to 7 . This was designed to allow participants in each trial to choose the exact rating that they wanted and not be limited by what was available from the random draw. We decided to use this structure to simplify the detection of acrophily. The order of ratings was randomized to make sure that participants looked at the whole screen. Here again, participants had to watch all the boxes for 5 seconds before making their pick. Second, instead of choosing to eliminate peers, participants chose the peer they wanted to keep. This was to make sure that acrophily can be detected both in decisions to keep and to eliminate. Third, participants were asked to choose just one peer of the seven on the screen. This was 
modified because there was always one peer per rating. Fourth, peers who were kept in a certain trial were not necessarily carried forward to the next trial due to the structure of the task. Unlike previous tasks, participants were not told explicitly that choosing to keep certain people would mean that participants would see them in subsequent trials.

Policy and Peer Pool. We conducted a pilot study to create the policy pool for the study, testing multiple six candidate policies. One hundred and six participants were presented with each policy and were asked to first provide a text response to the picture. Participants were then asked to rate their support for the policy using the same 1-7 scale that was used in the task. We selected the four policies that produced the least polarized ratings (to allow for the possibility of acrophily), with the intention of selecting two eliciting more support among conservatives and two among liberals: ("Gradually increasing military budget", "Reducing the power of the federal government over states", "Increasing affirmative action to minorities”, “Increasing restrictions on hunting”). As expected, our policies were rated differently by conservatives and liberals (see Supplementary Figure 3 in SI).

For each policy and for each rating (from 1-7), we chose 4 responses based on coherence and fit between the text and the ratings (see Supplementary Table 15 SI). In the cases in which it was possible, we tried to get two responses by a liberal and two responses by a conservative for each rating. In the few cases in which such responses were not available, we used responses from the other political group. This meant that participants in our task saw responses that were mostly produced by people who identified with their own political group, despite the fact that these included all ratings from 1-7 in response to each policy. 
Measures. While completing the task, we collected participants' ratings and text in response to each picture. We also recorded which peers they chose to keep versus eliminate. After completing the task, participants completed a survey which included a few questions, similar to Study 2, in which we examined potential motivations for acrophily (choosing people because they are more creative, provided new interpretations, made the participants feel good, etc.), a personality scale, as well as participants' political identification (see Supplementary Table 16-17 in SI for further analysis).

\section{Data availability:}

Data for study 1 is available here: https://osf.io/nz4dk/

Data for Studies 2-4 is available here: https://osf.io/649fq/

Data for the ABM is available here: https://osf.io/ad7vh/

\section{Code availability:}

Code for study 1 is available here: https://osf.io/nz4dk/

Code for Studies 2-4 is available here: https://osf.io/649fq/

Code for the ABM is available here: https://osf.io/ad7vh/

\section{Acknowledgements:}

The authors wish to thank Maria Reyes, Naila Ebeid, and Nathanial Hunt for their assistance in running the lab studies, Vibha Puri for her assistance in developing the simulations and processing the peer information, and Andrei Boutyline and Jillian Jordan for their comments on the research.

JS is supported in part by the German Academic Scholarship Foundation (Promotionsförderung der Studienstiftung des deutschen Volkes). The funder had no role in 
study design, data collection and analysis, decision to publish or preparation of the manuscript.

\section{Author Contribution Statement:}

AG, JJG, EH, RW and JMA conceived and designed the experiments. AG, ZH, JS and DB ran the experiments. AG and JMA analyzed Studies 1-4. AG wrote the paper and EH, RW, JMA and JJG were involved in reviewing and editing the manuscript.

\section{Competing Interests Statement:}

The authors declare no competing interests.

Figure 1. Three hypothetical tie selection strategies. The red ticker represents a chooser's moral outrage to a certain case of police brutality. The blue tickers represent potential ties' moral outrage, and the numbers represent the order of choosing. When applying homophily (A), the chooser elects to keep others whose responses are closest, regardless of whether they are more or less intense. When applying homophily + acrophily, the chooser has a preference for high outrage responses (B). When applying acrophily (C), the chooser keeps only others who are expressing higher outrage.

Figure 2. An outline of a trial in the choice condition. After being presented with a picture of police brutality against a Black demonstrator, participants were asked to: (1) provide a text response to the picture; (2) rate their negative emotions from 1-no emotion to 9-a lot of emotion; and (3) view 6 peer responses to the same picture (each response includes a name, a rating, and a text response) and choose 3 peers they do not wish to see in subsequent trials. The three peers that participants chose to keep stay for the next trial, and participants are able to see their responses to the following image. An enlarged version of a representative peer response is shown in panel 4 .

Figure 3. Results from Study $2(\mathrm{n}=213$, experimental condition). Panel A represents the acrophily coefficient (reverse scaled for conservatives for ease of comparison) over trial number, where positive values represent a tendency for acrophily. Error bars represent 95\% confidence intervals. Panel B represents a summary of comparisons between the average kept ratings and the four simulations (acrophily higher, acrophily lower, homophily, random). Each simulation was repeated 1,00o times and was compared to the actual average kept ratings. The zero line represents the average of participants' kept rating. Each red dot represents the average b coefficient for the simulation and its distribution. Participants' 
average kept ratings are located in between a pure homophily tie selection strategy and a pure acrophily strategy, higher for liberals and lower for conservatives.

Figure 4. Results from Study 3. The difference between participants' ratings and the peers they chose as most prototypical of their political camp in a rank order of 1 to $3(\mathrm{~N}=387)$. Liberals evaluated the first most prototypical peer as well as the second most prototypical peer to be expressing stronger emotions than their own emotions. The third peer seems to be equal to participants' emotions, but this is caused by the fact that there were not available peers who were expressing stronger emotions after the first two. Conservatives show the same patter but in the opposite direction. Conservatives evaluated the first most prototypical peer as well as the second most prototypical peer to be expressing less strong emotions compared to their own emotion. The third most prototypical peer seems to be expressing stronger emotions, but this is caused by the fact that there were not available peers who were expressing weaker emotions after the first two. Error bars represent 95\% confidence intervals.

Figure 5. Task and results of Study 4. Panel A provides a representative peer rating window from which participants were asked to choose one peer they would like to keep. Panel B represents the acrophily difference between participants and the peer they chose to keep in response to each concept $(\mathrm{N}=386)$. Positive values represent acrophily and results suggest acrophily in both the liberal and Conservative users. The boxes represent the interquartile range (IQR). The lines represent the first quartile $-1.5 \mathrm{IQR}$ and the third quartile + 1.5 IQR.

\section{References}

1. Brown, J. R. \& Enos, R. D. The measurement of partisan sorting for 180 million voters. Nat. Hum. Behav. (2021) doi:10.1038/s41562-021-01066-z.

2. Finkel, E. J. et al. Political sectarianism in America. Science 370, 533-536 (2020).

3. Bishop, B. The big sort: Why the clustering of like-minded America is tearing us apart. (Mariner Books, 2009).

4. Iyengar, S., Lelkes, Y., Levendusky, M., Malhotra, N. \& Westwood, S. J. The origins and consequences of affective polarization in the United States. Annu. Rev. Polit. Sci. 22, 135 (2018).

5. McPherson, M., Smith-Llovin, L. \& Cook, J. M. Birds of a feather: Homophily in social 
networks. Annu. Rev. Sociol. 27, 415-444 (2001).

6. Dehghani, M. et al. Purity homophily in social networks. J. Exp. Psychol. Gen. (2016).

7. Kossinets, G. \& Watts, D. J. Origins of homophily in an evolving social network. Am. J. Sociol. 115, 405-450 (2009).

8. Halberstam, Y. \& Knight, B. Homophily, group size, and the diffusion of political information in social networks: Evidence from Twitter. J. Public Econ. 143, 73-88 (2016).

9. Huber, G. A. \& Malhotra, N. Political homophily in social relationships: Evidence from online dating behavior. J. Polit. 79, 269-283 (2017).

10. Diprete, T. A., Gelman, A., Mccormick, T., Teitler, J. \& Zheng, T. Segregation in social networks based on acquaintanceship and trust . Am. J. Sociol. 116, 1234-83 (2011).

11. Brady, W. J., Wills, J. A., Jost, J. T., Tucker, J. A. \& Van Bavel, J. J. Emotion shapes the diffusion of moralized content in social networks. Proc. Natl. Acad. Sci. 114, 7313-7318 (2017).

12. Boutyline, A. \& Willer, R. The social structure of political echo chambers: Variation in ideological homophily in online networks. Polit. Psychol. 38, 551-569 (2017).

13. Morrison, K. R. \& Miller, D. T. Distinguishing between silent and vocal minorities: not all deviants feel marginal. J. Pers. Soc. Psychol. 94, 871-82 (2008).

14. Abrams, D., Bown, N., Marques, J. M. \& Henson, M. Pro-norm and anti-norm deviance within and between groups. J. Pers. Soc. Psychol. 78, 906-912 (2000).

15. Abrams, D., Marques, J., Bown, N. \& Dougill, M. Anti-norm and Pro-norm deviance in the bank and on the campus: Two experiments on subjective group dynamics. Gr. 
Process. Intergr. Relations 5, 163-182 (2002).

16. Kulibert, D., Moss, A., Appleby, J. \& O’brien, L. Perceptions of political deviants: A lay theory of subjective group dynamics. (2021) doi:10.31234/osf.io/aq652.

17. Zimmerman, F., Garbulsky, G., Ariely, D., Sigman, M. \& Navajas, J. The nonreciprocal and polarizing nature of interpersonal attraction in political discussions. 1-37 (2021) doi:10.31234/osf.io/b9645.

18. Goldenberg, A., Sweeny, T. D., Shpigel, E. \& Gross, J. J. Is this my group or not? The role of ensemble coding of emotional expressions in group categorization. J. Exp. Psychol. Gen. (2019) doi:10.1037/xgeoooo651.

19. Hogg, M. A. \& Adelman, J. Uncertainty-Identity Theory: Extreme Groups, Radical Behavior, and Authoritarian Leadership. J. Soc. Issues 69, 436-454 (2013).

20. Rabinowitz, G. \& Macdonals, S. E. A directional theory of issue voting. Am. Polit. Sci. Rev. 83, 93-121 (1989).

21. Bischof, D. \& Wagner, M. Do voters polarize when radical parties enter parliament? Am. J. Pol. Sci. 63, 888-904 (2019).

22. Tomz, M. \& Van Houweling, R. P. Candidate positioning and voter choice. Am. Polit. Sci. Rev. 102, 303-318 (2008).

23. Lacy, D. \& Paolino, P. Testing proximity versus directional voting using experiments. Elect. Stud. 29, 460-471 (2010).

24. Gallati, L. \& Giger, N. Proximity and directional voting: Testing for the region of acceptability. Elect. Stud. 64, 102024 (2020).

25. Dion, K. L., Baron, R. S. \& Miller, N. Why do groups make riskier decisions than 
individuals? Adv. Exp. Soc. Psychol. 5, 305-377 (1970).

26. Cartwright, D. Determinants of scientific progress: The case of research on the risky shift. Am. Psychol. 28, 222-231 (1973).

27. Moscovici, S. \& Zavalloni, M. The group as a polarizer of attitudes. J. Pers. Soc. Psychol. 12, 125-135 (1969).

28. Myers, D. G. \& Bishop, G. Discussion effects on racial attitudes. ScienceScience 169, 778-779 (1970).

29. Lord, C. G., Ross, L. \& Lepper, M. R. Biased assimilation and attitude polarization: The effects of prior theories on subsequently considered evidence. J. Pers. Soc. Psychol. 37, 2098-2109 (1979).

30. Myers, D. G. \& Lamm, H. The group polarization phenomenon. Psychol. Bull. 83, 602$627(1976)$.

31. Westfall, J., Judd, C. M. \& Kenny, D. A. Replicating studies in which samples of participants respond to samples of stimuli. Perspect. Psychol. Sci. 10, 390-399 (2015).

32. Skinner, M. \& Stephenson, G. M. The effects of intergroup comparison on the polarization of opinions. Curr. Psychol. Res. 1, 49-59 (1981).

33. Packer, D. J. On being both with us and against us: A normative conflict model of dissent in social groups. Personal. Soc. Psychol. Rev. 12, 50-72 (2008).

34. Jetten, J. \& Hornsey, M. J. Deviance and dissent in groups. Annu. Rev. Psychol. 65, 461485 (2014).

35. Hornsey, M. J. \& Jetten, J. The individual within the group: Balancing the need to belong with the need to be different. Personal. Soc. Psychol. Rev. 8, 220-247 (2004). 
36. Goldenberg, A. et al. Beyond emotional similarity: The role of situation specific motives. J. Exp. Psychol. Gen. 149, 138-159 (2019).

37. Robinson, R. J., Keltner, D., Ward, A. \& Ross, L. Actual versus assumed differences in construal: 'Naive realism' in intergroup perception and conflict. J. Pers. Soc. Psychol. 68, 404-417 (1995).

38. Ahler, D. J. \& Sood, G. The parties in our heads: Misperceptions about party composition and their consequences. J. Polit. 8o, 964-981 (2018).

39. Levendusky, M. S. \& Malhotra, N. (MIS)perceptions of partisan polarization in the American public. Public Opin. Q. 8o, 378-391 (2016).

40. Van Boven, L., Ehret, P. J. \& Sherman, D. K. Psychological Barriers to Bipartisan Public Support for Climate Policy. Perspect. Psychol. Sci. 13, 492-507 (2018).

41. Lau, T., Morewedge, C. K. \& Cikara, M. Overcorrection for social-categorization information moderates impact bias in affective forecasting. Psychol. Sci. 0956797616660292 (2016) doi:10.1177/095679761666o292.

42. Koller, M. robustlmm: An R package for robust estimation of linear mixed-effects models. J. Stat. Softw. 75, 1-24 (2016).

43. Batson, C. D. et al. Anger at unfairness: Is it moral outrage? Eur. J. Soc. Psychol. 37, 1272-1285 (2007).

44. Brady, W. J., Crockett, M. \& Bavel, J. J. Van. The MAD model of moral contagion: The role of motivation, attention and design in the spread of moralized content online. (2019) doi:10.31234/OSF.IO/PZ9G6.

45. Jordan, J. J. \& Rand, D. G. Signaling when no one is watching: A reputation heuristics 
account of outrage and punishment in one-shot anonymous interactions. J. Pers. Soc. Psychol. (2020) doi:10.1037/pspioooo186.supp.

46. van Kleef, G. A. How emotions regulate social life the emotions as social information (EASI) model. Curr. Dir. Psychol. Sci. 18, 184-188 (2009).

47. Panda, A., Siddarth, D. \& Pal, J. COVID, BLM, and the polarization of US politicians on Twitter. arXiv Prepr. arXiv2008.03263 (2020).

48. Costello, T. H. \& Bowes, S. M. Absolute Certainty and Political Ideology: A Systematic Test of Curvilinearity. Soc. Psychol. Personal. Sci. 194855062110704 (2022) doi:10.1177/19485506211070410.

49. van Prooijen, J. W. \& Krouwel, A. P. M. Psychological features of extreme political ideologies. Current Directions in Psychological Science vol. 28 159-163 (2019).

50. Van Der Does, T., Galesic, M., Dunivin, Z. O. \& Smaldino, P. E. Strategic identity signaling in heterogeneous networks. Proc. Natl. Acad. Sci. U. S. A. 119, 1-10 (2022).

51. Schweighofer, S., Schweitzer, F. \& Garcia, D. A weighted balance model of opinion hyperpolarization. J. Artif. Soc. Soc. Simul. 23, 1 (2020).

52. Roccas, S., Klar, Y. \& Liviatan, I. The paradox of group-based guilt: Modes of national identification, conflict vehemence, and reactions to the in-group's moral violations. J. Pers. Soc. Psychol. 91, 698-711 (2006).

53. Leary, M. R., Kelly, K. M., Cottrell, C. A. \& Schreindorfer, L. S. Construct validity of the need to belong scale: Mapping the nomological network. J. Pers. Assess. 95, 610-624 (2013).

54. Gosling, S. D., Rentfrow, P. J. \& Swann, W. B. A very brief measure of the Big-Five 
personality domains. J. Res. Pers. 37, 504-528 (2003).

55. Haddock, G., Zanna, M. P. \& Esses, V. M. Assessing the structure of prejudicial attitudes: The case of attitudes toward homosexuals. J. Pers. Soc. Psychol. 65, 1105-1118 (1993). 